\title{
Numération des cellules somatiques du lait cru par la technique DEFT associée à un comptage visuel ou par analyse d'image
}

\author{
A. Dasen, C. Piton, E. Beuvier * et R. Grappin
}

INRA, Station de recherches en technologie et analyses laitières, BP 94, 39800 Poligny, France

(reçu le 28 décembre 1988, accepté le 10 juillet 1989)

Résumé - La technique DEFT (direct epifluorescent filter technique), qui utilise la filtration sur membrane de porosité $1 \mu \mathrm{m}$ et la microscopie en épifluorescence, a été employée pour le dénombrement des cellules somatiques du lait cru. Quatre-vingt-six échantillons de lait cru contenant entre $1,2.10^{4}$ et $5,5.10^{6}$ cellules somatiques $/ \mathrm{ml}$ ont été analysés en double par la méthode microscopique de référence, la technique DEFT et l'appareil automatique Fossomatic. L'écart type résiduel de la régression référence sur DEFT est de $0,082 \log _{10}$ cellules/ml; pour la regression référence sur Fossomatic, il est de 0,078 . Par rapport à la méthode de référence, la technique DEFT sous-estime les nombres de cellules somatiques en moyenne de $4 \%$. L'écart type relatif supérieur de répétabilité (RSD+) est, en moyenne, de $21,3 \%$ pour la DEFT; il est de $14,8 \%$ pour la méthode microscopique de référence et de $5,4 \%$ pour le Fossomatic. Pour les techniques microscopiques, le rôle très important des comptages sur les résultats de dénombrement permet d'expliquer les valeurs élevées du RSD+ de répétabilité, particulièrement pour les faibles concentrations cellulaires. L'écart type relatif supérieur de reproductibilité, à l'intérieur d'un même laboratoire, de la DEFT est de $23,5 \%$. Cent quatre-vingt-quatre membranes DEFT ont été comptées à la fois à l'œil nu et par l'analyseur d'image 4010 (Foss Electric). Après transformation racine carrée des données, le coefficient de corrélation obtenu entre les 2 comptages est de 0,982 et l'écart type résiduel de la régression comptage visuel

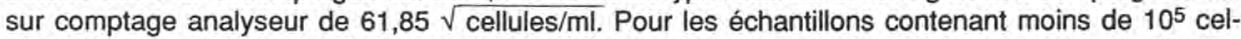
lules/ml, les nombres de cellules obtenus par analyse d'image sont significativement plus élevés que ceux de comptage visuel, probablement en raison du comptage de débris colorés. A partir de 4 jours de stockage des échantillons de lait à $4^{\circ} \mathrm{C}$, nous avons mis en évidence une baisse significative des nombres de cellules obtenus par la DEFT; à l'inverse, l'addition de conservateurs (dichromate de potassium, formol ou bronopol) permet de conserver les échantillons à $4{ }^{\circ} \mathrm{C}$ pendant 15 jours, sans observer de baisse significative de ces nombres.

lait cru — numération leucocytaire — DEFT — précision — fidélité — analyse d'image

Summary - Enumeration of the somatic cells in raw milk, using the Direct Epifluorescent Filter Technique (DEFT) with a visual or an image analyser counting. The Direct Epifluorescent Filter Technique (DEFT) which associates the membrane filtration technique and the epifluorescent microscopy has been used for the enumeration of somatic cells in raw milk. A series of 86 raw milk samples, ranging from $1.2 \times 10^{4}$ to $5.5 \times 10^{6}$ somatic cells $/ \mathrm{ml}$ was analysed in duplicate by the direct

\footnotetext{
*Etudiant DESS microbiologie, Université Claude Bernard (Lyon I), 69000 Lyon.
} 
microscopic reference method (DMRM), the DEFT and the automated instrument "Fossomatic". The accuracy expressed by the residual standard deviation from the regression DMRM on DEFT, is 0.082 $\log _{10}$ cells/ml; for the regression DMRM on Fossomatic, the residual standard deviation is 0.078 . The average DEFT count is significantly lower than the DMRM count by $4 \%$. The repeatability upper relative standard deviation (RSD+) of the DMRM and the DEFT averages resp. 14.8 and $21.3 \%$ and becomes proportionally higher as the count decreases; for the Fossomatic, it averages $5.4 \%$. The DMRM and DEFT counts are more affected by the counting than by the reader or the operator preparing the slides or the membranes. One hundred eighty four DEFT membranes were examined both visually and by the Image Analyser 4010 (Foss Electric). After a square root transformation of data, the coefficient of correlation between the two counting procedures is 0.982 and the residual standard deviation from the regression of the visual count over the semi-automated count is $61.85 \sqrt{\text { cells } / \mathrm{ml}}$. For the samples below $10^{5} \mathrm{cell} / \mathrm{s} / \mathrm{ml}$, the semi-automated DEFT cell counts are significantly higher than the visual DEFT counts, probably because of the small number of cells per field and the presence of coloured debris. The DEFT somatic cell counts decrease significantly after 4 days of storage of the milk samples at $4{ }^{\circ} \mathrm{C}$. Addition of preservatives $\left(\mathrm{K}_{2} \mathrm{Cr}_{2} \mathrm{O}_{7}\right.$, formalin and bronopol) allows the DEFT counts to remain constant during 15 days of storage at $4{ }^{\circ} \mathrm{C}$.

\section{raw milk - somatic cell count - DEFT - accuracy - precision - image analyser}

\section{INTRODUCTION}

La lutte contre les infections mammaires et les normes hygiéniques imposées au lait cru nécessitent un contrôle régulier de sa concentration en cellules somatiques au niveau de la production et de la transformation. Pour effectuer la numération des cellules somatiques, les laboratoires disposent maintenant d'appareils entièrement automatiques à cadence élevée et la fourniture de laits étalons à ces laboratoires a, par ailleurs, permị de réaliser un calibrage identique de tous ces appareils et d'obtenir une précision suffisante par rapport à la méthode microscopique de référence.

Mais compte tenu de leur coût élevé, seuls les laboratoires centraux regroupant un grand nombre d'échantillons peuvent s'équiper de ces appareils. Du fait de l'éloignement des unités de transformation de ces laboratoires, il est parfois impossible de connaître le résultat de l'analyse avant la transformation du lait. Les laboratoires d'usine sont alors contraints d'effectuer la numération des cellules somatiques par la méthode microscopique de référence (Anonyme, 1984, 1987). Mais cette technique présente l'inconvénient d'être délicate, fatigante pour l'opérateur et possède un seuil de détection élevé, compte tenu du facteur microscopique important.

La technique DEFT (ou direct epifluorescent filter technique), développée par Pettipher et al. (1980) pour le dénombrement de la flore totale du lait cru, a ensuite été adaptée pour la numération leucocytaire (Pettipher \& Rodrigues, 1981). Elle repose sur une concentration des cellules somatiques par filtration sur une membrane de façon à augmenter le seuil de détection de la technique, et une coloration des cellules à l'aide d'un fluorochrome pour faciliter le comptage au microscope.

En dehors des travaux originaux de Pettipher \& Rodrigues (1981), cette nouvelle méthode de numération des cellules somatiques du lait cru n'a fait l'objet d'aucune autre étude. Ces auteurs ont étudié sa fidélité mais sans préciser l'influence respective de l'opérateur, de la préparation de la membrane pour un même opérateur et du lecteur. D'autre part, ils ont établi sa 
justesse par rapport à une technique automatisée (compteur Coulter) et non pas par rapport à la technique microscopique de référence qui, par convention, donne la valeur vraie du nombre de cellules somatiques. Le premier objectif de notre étude a donc été de déterminer expérimentalement toutes les caractéristiques analytiques de cette technique (fidélité et justesse par rapport à la méthode de référence) et de les comparer à celles de l'appareil automatique Fossomatic.

La luminosité des cellules somatiques colorées par l'acridine orange provoque assez rapidement une fatigue de l'opérateur au microscope et limite la cadence de la technique à 30-40 échantillons/jour par opérateur, justifiant ainsi une automatisation du comptage (Pettipher \& Rodrigues, 1982). Dans une seconde partie, nous avons établi les caractéristiques analytiques d'un comptage des cellules somatiques par analyse d'image.

Enfin, la coloration rouge orangé des cellules par le fluorochrome étant étroitement liée à leur taux d'acide ribonucléique et à leur activité métabolique (Armstrong, 1956; Von Bertalanffy \& Bickis, 1956), nous avons étudié l'influence d'un stockage prolongé des échantillons de lait à $4{ }^{\circ} \mathrm{C}$ avec ou sans addition de conservateur sur les résultats de la technique DEFT.

\section{MATÉRIEL ET MÉTHODES}

\section{Techniques utilisées}

\section{Méthode microscopique de référence}

Les échantillons de lait cru ont été soumis au dénombrement des cellules somatiques selon la norme officielle (Anonyme, 1987). Le comptage des cellules a été effectué dans 50 champs répartis sur toute la surface de l'étalement; dans nos conditions expérimentales, le facteur microscopique était de 162000 .

\section{Fossomatic}

Chaque échantillon de lait a été analysé en double à l'aide de l'appareil automatique Fossomatic 215 décrit dans sa première version par Grappin \& Jeunet (1974). Cet appareil a été calibré à l'aide des laits étalons fournis régulièrement par notre station de recherches aux laboratoires d'analyses disposant d'appareils automatiques.

\section{Technique DEFT avec comptage visuel des cellules}

Nous avons utilisé la technique décrite par Pettipher \& Rodrigues (1981), en y apportant quelques modifications. Nous en rappelons ici les principales étapes.

\section{Préparation des membranes}

Un volume de $0,4 \mathrm{ml}$ d'une solution de formaldéhyde à $40 \%$ diluée au $1 / 10$ est ajouté à $10 \mathrm{ml}$ de lait. L'échantillon est ensuite dilué au 1/5 dans de l'eau distillée stérile; $1 \mathrm{ml}$ du lait dilué est mis à incuber à $80^{\circ} \mathrm{C}$ pendant $10 \mathrm{~min}$ dans un tube, de façon à durcir la membrane des cellules somatiques et éviter leur lyse au cours de la filtration. Après incubation, l'échantillon de lait dilué est additionné de $6 \mathrm{ml}$ d'une solution à $80^{\circ} \mathrm{C}$ de triton X 100 à la concentration de $0,1 \%$.

Une membrane en polycarbonate (Nuclepore) de $1 \mu \mathrm{m}$ de porosité et de $25 \mathrm{~mm}$ de diamètre est montée, côté brillant vers le haut, sur le système de filtration relié à une pompe à vide. La dépression appliquée est de 40 à $50 \mathrm{kPa}$; elle est annulée avant toute filtration, de façon à obtenir une bonne répartition et une coloration homogène des cellules sur toute la surface de la membrane. Après filtration de l'échantillon de lait dilué, le tube et le filtre sont rincés à l'aide de $6 \mathrm{ml}$ de la solution de triton $X 100$ préchauffée à $80^{\circ} \mathrm{C}$.

La coloration des cellules somatiques est ensuite effectuée par addition d'une solution d'acri- 
dine orange (RAL) à la concentration de $0,025 \%$ dans un tampon citrate $\mathrm{pH} 6,6$. Après un temps de contact de $4 \mathrm{~min}$, le colorant est éliminé et la membrane est rincée avec $2,5 \mathrm{ml}$ de la solution de triton $X 100$ puis avec $2,5 \mathrm{ml}$ d'isopropanol. Ces 2 rinçages sont effectués rapidement, de façon à éviter la décoloration des cellules somatiques. La membrane est ensuite séchée à l'air et disposée dans une immersion d'huile non fluorescente entre lame et lamelle.

Avant analyse, tous les réactifs sont filtrés sur membrane en acétate de cellulose de porosité $0,22 \mu \mathrm{m}$, de façon à assurer leur stérilisation et l'élimination des particules en suspension.

\section{Observation microscopique}

Les membranes ont été examinées le jour de leur préparation à l'aide d'un microscope Olympus BHS équipé d'un dispositif d'épifluorescence, d'un objectif S Plan achromatique 100X à immersion et d'un oculaire WHX $10 \mathrm{X}$ équipé d'un quadrillage. Le facteur de multiplication de ce microscope était, dans ces conditions expérimentales, de 55380 .

Les cellules colorées en orange ont été comptées dans des champs microscopiques pris au hasard, selon les normes indiquées dans le Tableau I.

Pour les membranes ayant plus de $50 \mathrm{cel}$ lules par champ, nous n'avons pas effectué de dilution de l'échantillon, comme le recommandent Pettipher \& Rodrigues (1981). Dans ce cas, nous avons examiné seulement la partie du champ délimitée par le quadrillage de l'oculaire et représentant $30 \%$ de la surface totale du champ; le nombre de champs est resté arbitrairement fixé à 10 .

\section{Technique DEFT avec comptage semi- automatique des cellules à l'aide d'un analyseur d'image}

Nous avons utilisé l'analyseur d'image 40-10 commercialisé par la société Foss Electric France. Le système comprend une caméra qui transmet l'image observée dans le microscope à l'analyseur d'image et à un écran vidéo qui permet à l'opérateur d'observer l'image transmise. L'analyseur compte les points lumineux par comparaison à une échelle de niveaux de gris; il est contrôlé par un microprocesseur qui traite les données fournies. Pour ce comptage, le microscope était équipé d'un objectif achromatique DA 60X à sec. Dans ces conditions expérimentales, le facteur de multiplication du système de comptage était de 55120.

Pour chaque membrane observée par l'analyseur d'image, nous avons effectué 2 comptages sur 15 champs choisis au hasard, quelle que soit la concentration cellulaire du lait.

\section{Protocole expérimental}

Pour la détermination des caractéristiques anaIytiques de la technique DEFT et de l'appareil Fossomatic, l'étude a porté sur 86 échantillons de lait cru; 56 provenaient du mélange du lait d'une seule traite, 15 de laits individuels et 16 de quartiers d'animaux infectés. La nature des échantillons nous a permis d'obtenir une gamme de concentrations variant de $1,2.10^{4}$ à $5,5.10^{6}$ cellules somatiques $/ \mathrm{ml}$. Les échantillons ont été conservés à $4{ }^{\circ} \mathrm{C}$ jusqu'au moment de l'analyse, au plus tard $12 \mathrm{~h}$ après la traite. Dans le cas de la numération à l'aide de l'appareil

Tableau I. Normes de comptage des cellules somatiques sur les membranes DEFT.

\begin{tabular}{cc}
$\begin{array}{c}\text { Nombres de champs } \\
\text { à compter }\end{array}$ & $\begin{array}{c}\text { Nombre moyen de cellules } \\
\text { par champ }\end{array}$ \\
\hline 15 & 0 à 10 \\
10 & 10 à 50 \\
10 & $>50$ \\
\hline
\end{tabular}




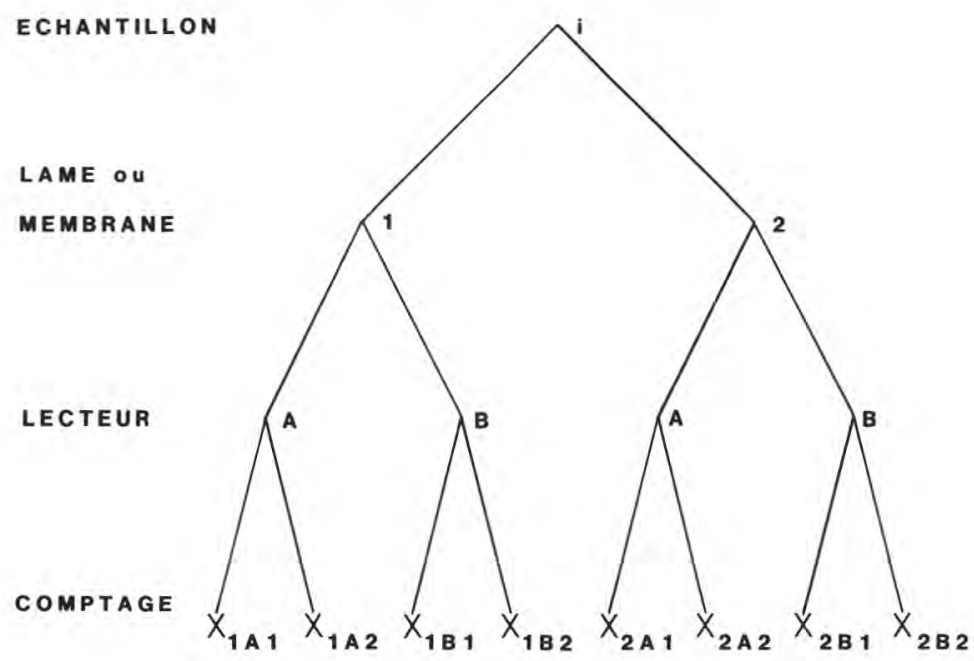

Fig. 1. Plan expérimental soumis à 86 échantillons de lait cru analysés selon la technique DEFT et la technique microscopique de référence.

Fossomatic, les échantillons ont été additionnés de dichromate de potassium à la dose de $1 \%$ et conservés à $4^{\circ} \mathrm{C}$ jusqu'à l'analyse, au plus tard 8 jours après le prélèvement.

Le dénombrement des cellules somatiques par l'appareil Fossomatic a été effectué sur les 86 échantillons, à 2 reprises dans un court laps de temps (moins de $15 \mathrm{~min}$ ). Pour la technique DEFT et la technique microscopique de référence, chaque échantillon i a été soumis au protocole décrit sur la Figure 1. Deux lames (ou membranes) ont été préparées par l'opérateur A et chaque lame (ou membrane) a été examinée à 2 reprises par 2 lecteurs $A$ et $B$, soit au total 2 lames $\times 2$ lecteurs $\times 2$ comptages $=8$ comptages par échantillon. D'autre part, pour apprécier également l'influence de l'opérateur sur les résultats de la technique DEFT, 26 des 86 échantillons ont été analysés par cette technique selon un protocole plus complexe : 2 opérateurs $A$ et $B$ ont préparé chacun 2 membranes ; ces 4 membranes DEFT ont ensuite été examinées à 2 reprises par ces 2 opérateurs $A$ et $B$, ce qui a donné un total de 16 comptages par échantillon.
L'étude du comptage automatique des cellules par analyse d'image a porté sur 184 échantillons de lait cru de diverses origines provenant du laboratoire d'analyses agricoles de Poligny. Les 184 membranes DEFT ont été examinées à 2 reprises le jour de leur préparation, visuellement par l'opérateur $A$ et automatiquement par l'analyseur d'image.

Enfin, pour étudier l'incidence d'une conservation prolongée du lait à $4^{\circ} \mathrm{C}$ sans addition de conservateur sur les résultats de la technique DEFT, 59 échantillons ont été prélevés au moment de la traite puis aussitôt répartis en 4 fractions de $30 \mathrm{ml}$. L'une de ces fractions a été analysée le jour même par la technique DEFT; les trois autres fractions l'ont été respectivement après 1,2 et 4 jours de conservation à $4^{\circ} \mathrm{C}$. Dans le cas de la conservation du lait avec addition de conservateurs, 30 échantillons ont été prélevés et divisés en 10 fractions de $30 \mathrm{ml}$. L'une de ces fractions était analysée le jour même et les 9 autres, additionnées d'un des 3 conservateurs suivants : dichromate de potassium $(1 \%)$, bronopol $(0,2 \%)$ ou formol $(1 \%)$, étaient analysées après 4,8 et 15 jours de stockage à $4^{\circ} \mathrm{C}$. 


\section{Calculs statistiques}

\section{Répétabilité et justesse}

Les caractéristiques analytiques des techniques expérimentales (répétabilité et justesse) ont été établies à partir des résultats des analyses en double, selon les recommandations de la norme FIL 128 (Anonyme, 1985) et après transformation logarithmique pour tenter de normaliser la distribution. Dans le cas de méthodes microscopiques, la répétabilité a d'abord été calculée à partir des résultats obtenus par l'opérateur A préparant 2 lames (ou membranes) pour chaque échantillon et effectuant un comptage par lame (ou membrane), c'est-à-dire les valeurs $\mathrm{x}_{1 \mathrm{~A} 1}$ et $\mathrm{X}_{2 \mathrm{~A} 1}$ sur la Figure 1. De même, ce sont ces 2 valeurs qui ont servi à l'établissement de la droite de calibrage.

Pour le calcul de cette droite, le logarithme décimal du nombre de cellules obtenu avec la technique de référence est considéré comme variable dépendante, pour donner une estimation de l'écart type résiduel $\left(s_{y, x}\right)$ dans la même unité que la technique de référence. La précision d'estimation de la technique expérimentale est donnée par les limites de l'intervalle de confiance à l'intérieur duquel se situe, avec une probabilité de $95 \%$, la vraie valeur donnée par la méthode de référence. Ces limites sont, en valeur logarithmique, $\pm 1,96 . s_{y, x}$ si $y$ est le nombre de cellules calculé à partir de la droite de calibrage, ces limites deviennent en données non transformées :

$y / 10^{1,965 x}$, y pour la limite inférieure et $y \cdot 10^{1,96 s x}$, y pour la limite supérieure.

Afin de pouvoir éliminer de l'estimation de l'écart type résiduel l'influence de l'erreur de mesure de la méthode de référence, la droite de calibrage a été calculée à partir de la moyenne des 2 déterminations faites sur chaque échantillon $i$ par la technique expérimentale $\left(\overline{x_{i}}\right)$, et chacun des 2 résultats individuels obtenus avec la

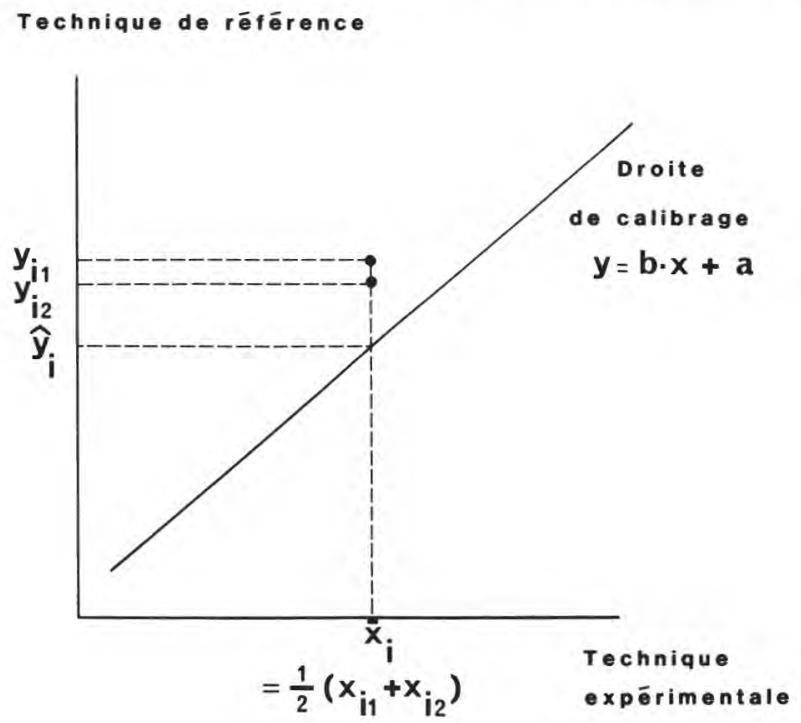

Fig. 2. Mode de calcul de la droite de calibrage des techniques expérimentales. 
technique de référence sur cet échantillon $\left(y_{i 1}\right.$ et $\left.y_{i 2}\right)$, comme le montre la Figure 2. Une analyse de variance à une voie a ensuite été effectuée sur les résidus, $d_{i j}=y_{i j}$ $-\hat{y}_{i}, y_{i j}$ étant l'une des 2 valeurs observées avec la méthode de référence sur l'échantillon $i$ et $\hat{y}_{i}$ la valeur prédite pour l'échantillon i à partir de la moyenne des 2 déterminations effectuées par la technique expérimentale $\left(\bar{x}_{i}\right)$. Les résultats de cette analyse permettent d'estimer l'écart type de répétabilité de la technique de référence $\left(s_{r}\right)$ et l'écart type résiduel «réel» $\left(s_{y}, x^{\prime}\right)$, déduction faite de l'incertitude de mesure de la méthode de référence (Tableau II). Les graphes ont été construits à partir des moyennes des deux déterminations effectuées par la technique de référence et la technique expérimentale.

\section{Influence respective de l'opérateur, de la lame (ou membrane) et du lecteur sur les résultats des techniques microsco- piques}

Pour la technique de référence et la technique DEFT, les 8 résultats de dénombrement obtenus sur chacun des 86 échantillons (Fig. 1) ont été soumis à une analyse de variance à modèle croisé hié- rarchisé puisqu'il n'existe aucune correspondance entre les 2 lames ou membranes préparées sur les différents échantillons (Dagnelie, 1970). Les facteurs pris en compte dans le modèle sont les suivants : échantillon, lame (ou membrane), lecteur et répétition du comptage. Les résultats de cette analyse permettent d'estimer la variance inter-lames ou intermembranes $\left(s_{L}{ }^{2}\right)$, la variance inter-lecteurs $\left(\mathrm{s}^{2}\right)$ ainsi que la variance de répétabilité du comptage $\left(\mathrm{s}_{\mathrm{C}}{ }^{2}\right)$ (Tableau IIla, d'après Dagnelie (1970)).

De même, pour estimer l'influence de l'opérateur sur les résultats de la technique DEFT, les 16 résultats de dénombrement par échantillon obtenus sur 26 échantillons (cf. "Protocole expérimental») ont été soumis à une analyse de variance à modèle croisé hiérarchisé. Les facteurs pris en compte sont alors les suivants : échantillon, opérateur, interaction échantillon $x$ opérateur, membrane, lecteur et répétition du comptage. Ce modèle permet d'estimer la variance interopérateurs $\left(\mathrm{s}_{\mathrm{O}}{ }^{2}\right)$ ainsi que celle de l'interaction échantillon $x$ opérateur $\left(s_{\mathrm{ExO}} \mathrm{O}^{2}\right)$ (Tableau IIl b).

A partir de ces variances estimées, il est possible de calculer les variances de répétabilité des 2 techniques $\left(s_{\mathrm{r}}{ }^{2}\right)$, sur la base de la préparation d'une seule lame

Tableau II. Calcul de la précision d'estimation des techniques expérimentales.

Source de variation

$d d l$

Estimation des variances

\begin{tabular}{lll}
\hline Echantillon & $n-1$ & $s_{r}^{2}+2 s_{y, x^{2}}$ \\
Répétition & $n$ & $s_{r}^{2}$ \\
\hline
\end{tabular}

Analyse de variance des résidus $d_{j j}$ de la régression $y_{i j}=b \bar{x}_{i}+a ; y_{i j}$ : résultats individuels obtenus pour l'échantillon i selon la méthode de référence; $\bar{x}_{i}:$ moyenne des 2 déterminations effectuées pour l'échantillon $i$ selon la technique expérimentale. 
Tableau III. a. Analyse de variance des résultats obtenus pour $n$ échantillons avec 8 résultats par échantillon ( 2 lames ou membranes $\times 2$ lecteurs $\times 2$ comptages). b. Analyse de variance des résultats obtenus pour $n$ échantillons avec 16 résultats par échantillon ( 2 opérateurs $\times 2$ membranes $\times 2$ lecteurs $\times 2$ comptages).

Tableau a.

\begin{tabular}{lll}
\hline Source de variation & $d d l$ & Estimation des variances \\
\hline Echantillon (n) & $n-1$ & \\
Lame (membrane) (2) & $n$ & $s_{\mathrm{C}}^{2}+4 s_{\mathrm{L}}^{2}$ \\
Lecteur (2) & 1 & $s_{\mathrm{C}}^{2}+4 n s s^{2}$ \\
Comptage (2) & & $s_{\mathrm{C}}^{2}$ \\
\hline
\end{tabular}

Tableau b.

\begin{tabular}{|c|c|c|}
\hline Source de variation & $d d l$ & Estimation des variances \\
\hline Echantillon ( $n$ ) & $n-1$ & \\
\hline Opérateur (2) & 1 & $s_{C^{2}}^{2}+4 s_{L}^{2}+8 s_{E \times O^{2}}+8 n s_{O}^{2}$ \\
\hline $\begin{array}{l}\text { Echantillon } \\
\text { x opérateur }\end{array}$ & $n-1$ & $s_{C^{2}}+8 s_{E \times O^{2}}$ \\
\hline Membrane (2) & $2 n$ & $s_{C}^{2}+4 s_{L}^{2}$ \\
\hline Lecteur (2) & 1 & $s_{C^{2}}^{2}+8 \pi s^{2}$ \\
\hline Comptage (2) & & $s_{c}^{2}$ \\
\hline
\end{tabular}

(ou membrane) par échantillon et d'un seul comptage par lame, grâce à la formule :

$$
s_{r}^{2}=s_{C}^{2}+s_{L}^{2}
$$

De même, il est possible d'estimer la variance de reproductibilité, à l'intérieur d'un même laboratoire, de la technique DEFT, bien que les analyses aient été effectuées sur des échantillons uniques; cette variance est égale à :

$$
s_{\mathrm{R}}^{2}=s_{\mathrm{C}}{ }^{2}+s_{\mathrm{L}}^{2}+s_{\mathrm{O}}^{2}+s_{E \times \mathrm{O}^{2}}
$$

Enfin, on peut calculer, à partir des écarts types de répétabilité $s$ (en $\log _{10}$ ), les écarts types relatifs supérieurs RSD+ en données non transformées à partir de la formule suivante :

$$
\mathrm{RSD}^{+}=\left(10^{\mathrm{S}}-1\right) \cdot 100
$$

\section{RÉSULTATS}

\section{Fidélité}

Le Tableau IV compare la répétabilité de la technique DEFT à celles de la technique microscopique de référence et de l'appareil Fossomatic. On observe tout d'abord que l'écart-type de répétabilité de l'appareil Fossomatic est plus faible que celui des techniques microscopiques, quelle que soit la concentration cellulaire du lait. D'autre part, les calculs effectués par niveau montrent une nette augmentation de cet écart type pour les méthodes 
microscopiques lorsque la concentration cellulaire du lait baisse. L'écart type de répétabilité de la technique DEFT est significativement plus élevé (à $P<0,05$ ) que celui de la méthode de référence audessous de 7,5.105 cellules/ml (test $\mathrm{F}$ de comparaison des variances) (Dagnelie, 1970). II en résulte que, pour l'ensemble des échantillons, le RSD+ de la DEFT est en moyenne significativement plus élevé que celui des 2 autres techniques.

Le Tableau $V$ permet d'estimer la part des différents facteurs dans la variance des résultats de la technique DEFT et de calculer les variances de répétabilité et de reproductibilité de cette technique. On observe que les variances les plus élevées sont de loin celles de la répétition des comptages et de l'interaction échantillon $x$ opérateur. A titre de comparaison, l'effet lames n'était pas significatif pour la technique de référence et les variances de répétabilité dues au lecteur et au comptage étaient respectivement de $1,28.10^{-4}$ et de 3,466.10-3. La variance de répétabilité de la DEFT est donc de $5,815.10^{-3}$, ce qui

Tableau IV. Répétabilité de la technique DEFT, de la technique microscopique de référence et du Fossomatic pour la numération des cellules somatiques du lait cru.

\begin{tabular}{|c|c|c|c|c|c|c|c|c|}
\hline \multirow{2}{*}{$\begin{array}{l}\text { Nombre de } \\
\text { cellules } / \mathrm{ml}\end{array}$} & \multicolumn{3}{|c|}{ Moyenne $\left(x 10^{3}\right)$} & $E F T$ & \multicolumn{2}{|c|}{ Référence } & \multicolumn{2}{|c|}{ Fossomatic } \\
\hline & $N$ & & $\mathrm{~s}_{\mathrm{r}}$ & $R S D^{+}$ & $s_{\mathrm{r}}$ & $R S D^{+}$ & $s_{r}$ & $R S D^{+}$ \\
\hline Moins de $2,5.10^{5}$ & 26 & 126 & 0,135 & 36,0 & 0,092 & 23,5 & 0,034 & 8,1 \\
\hline $2,5-7,5.10^{5}$ & 23 & 482 & 0,051 & 12,4 & 0,012 & 2,8 & 0,019 & 4,4 \\
\hline Plus de $7,5,10^{5}$ & 37 & 1800 & 0,039 & 9,4 & 0,033 & 7,8 & 0,013 & 3,0 \\
\hline Total & 86 & 480 & 0,084 & 21,3 & 0,060 & 14,8 & 0,023 & 5,4 \\
\hline
\end{tabular}

$\mathrm{N}$ : nombre d'échantillons; $s_{\mathrm{r}}$ : écart type de répétabilité (en $\left.\log _{10}\right) ; \mathrm{RSD}^{+}$: écart type relatif supérieur de répétabilité (en données non transformées) calculé à partir de $\mathrm{s}_{\mathrm{r}}$.

Tableau V. Fidélité de la technique DEFT.

\begin{tabular}{lcr}
\hline $\begin{array}{c}\text { Source de } \\
\text { variation }\end{array}$ & $\begin{array}{c}\text { Variance estimée } \\
\mathrm{s}^{2}\left(e n \log _{10}\right)\end{array}$ \\
\hline Opérateur & 0,000378 & 4,6 \\
Echantillon x opérateur & 0,002155 & 11,3 \\
Lame & 0,000797 & 6,7 \\
Lecteur & 0,000044 & 1,5 \\
Comptage & 0,005018 & 17,7 \\
\hline
\end{tabular}

$\mathrm{RSD}^{+}$: écart type relatif supérieur de répétabilité (en données non transformées) calculé à partir de $s$. 
correspond à un RSD+ de 19,2\%; cette variance est légèrement différente de celle présentée sur le Tableau II car dans ce premier cas, elle avait été estimée à partir de 2 des résultats obtenus par l'opérateur $A\left(x_{1 A 1}\right.$ et $X_{2 A 1}$ sur la Figure 1). La variance de répétabilité de la DEFT est significativement supérieure (à $P<0,05)$ à celle de la technique de référence $\left(3,466 \cdot 10^{-3}\right)$. D'autre part, la variance de reproductibilité, à l'intérieur d'un même laboratoire, de la technique DEFT $\left(\mathrm{s}_{\mathrm{R}}{ }^{2}\right)$ est égale à 8,392.10-3; l'écart type relatif supérieur de reproductibilité à l'intérieur d'un même laboratoire est donc de $23,5 \%$.

\section{Justesse}

La Figure 3 montre la relation entre les logarithmes des nombres de cellules $/ \mathrm{ml}$ de lait obtenus avec la technique microscopique de référence d'une part et la technique DEFT d'autre part; cette relation a été établie à partir d'une population de 86 échantillons. La précision d'estimation de cette technique est de $\pm 1,96 \times 0,070$, soit $\pm 0,137 \log _{10}$ cellules $/ \mathrm{ml}$. Si $y$ est le nombre de cellules $/ \mathrm{ml}$ calculé à partir de la droite de calibrage, les limites de l'intervalle de confiance de y (à $P=0,95)$ sont en données non transformées :

\section{y / 1,37 pour la limite inférieure} et $y \times 1,37$ pour la limite supérieure.

Cependant, on observe que la dispersion des points autour de la droite de régression devient plus importante pour les échantillons contenant moins de 105 cellules somatiques $/ \mathrm{ml}$. La suppression de ces 9 échantillons dans le calcul de la droite de calibrage permet d'améliorer significativement la précision d'estimation de la technique (test $\mathrm{F}$ de comparaison des variances) (Dagnelie, 1970); en revanche,cela ne modifie pas de façon significative l'équation de la droite de calibrage qui devient :

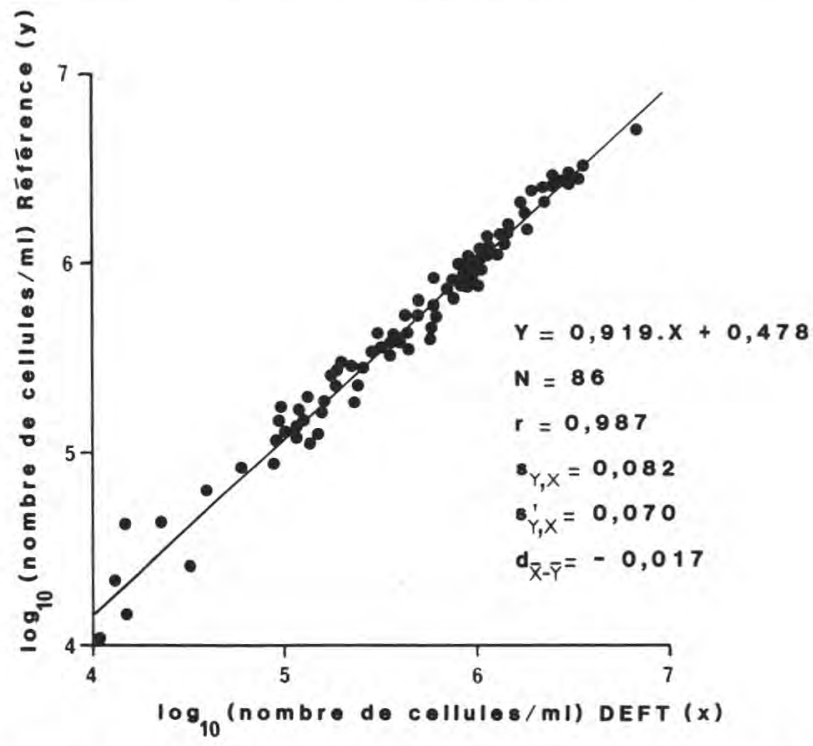

Fig. 3. Relation entre les résultats de dénombrement des cellules somatiques du lait cru par la technique DEFT et par la technique microscopique de référence. 


$$
y=0,914 x+0,506
$$

avec $s_{y, x}=0,066 ; s_{y, x^{\prime}}=0,056$ et $d_{\bar{x}-\bar{y}}=-0,006$.

Avec l'appareil Fossomatic, l'équation de calibrage obtenue à partir de l'analyse de la même population de 86 échantillons est :

$$
y=1,037 x-0,237
$$

avec $s_{y, x}=0,078 ; s_{y, x^{\prime}}=0,066$ et $d_{\bar{x}-\bar{y}}=+0,025$.

La précision d'estimation de la DEFT ne diffère donc pas significativement (à $\mathrm{P}<$ $0,05)$ de celle de l'appareil automatique Fossomatic (test $\mathrm{F}$ de comparaison des variances; Dagnelie, 1970).

Avec une erreur standard sb de 0,012, le coefficient de régression de la droite de calibrage est significativement inférieur à 1,000 dans le cas de la technique DEFT; d'autre part, les deux moyennes log (Référence) $=5,676$ et log (DEFT) $=5,658$ sont statistiquement différentes à $P<0,05$ (test t par paires; Dagnelie, 1970). Pour corriger les résultats de la DEFT du défaut de calibrage, il est nécessaire d'appliquer à ces résultats l'équation de calibrage donnée sur la figure 3 . Une autre manière consiste à appliquer un facteur de correction correspondant au rapport des moyennes des résultats des deux comptages; ce mode de calcul, plus simple mais moins rigoureux, conduit à des erreurs d'autant plus grandes que l'on s'éloigne de la moyenne. Dans ce cas, le facteur de correction est égal à 1,04 , ce qui signifie que les valeurs obtenues par la DEFT doivent être multi-

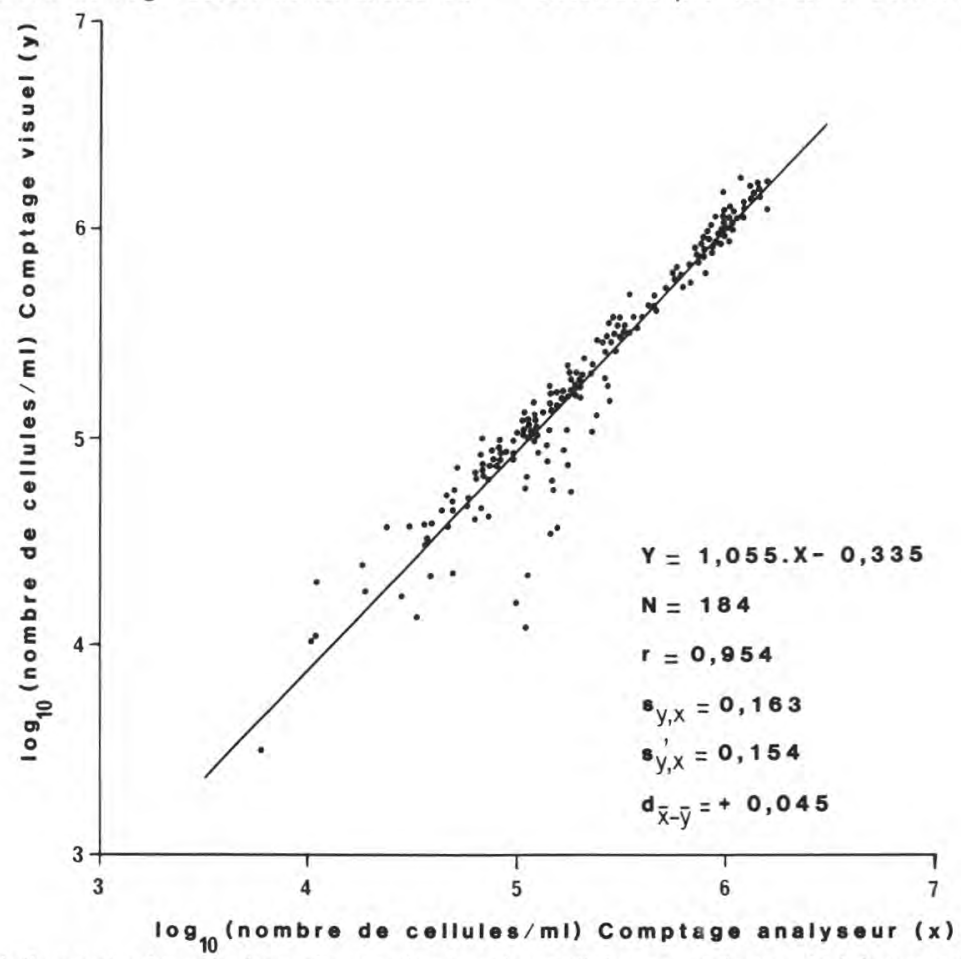

Fig. 4. Relation entre les résultats de numération des cellules somatiques du lait cru par la technique DEFT à l'aide d'un comptage par analyseur d'image et d'un comptage visuel, après transformation logarithmique des résultats. 
pliées par 1,04 pour avoir des résultats en moyenne égaux à ceux de la méthode de référence.

\section{Comptage des cellules sur membranes DEFT par analyse d'image}

La Figure 4 présente la relation obtenue pour 184 membranes DEFT entre les logarithmes des résultats du comptage visuel des cellules et par analyse d'image. On observe très nettement une dissymétrie de la distribution autour de la droite de régression, montrant que la transformation logarithmique des nombres de cellules n'est donc pas appropriée. Cela est probablement lié au fait que la distribution des cellules à la surface de la membrane suit approximativement une loi de Poisson pour laquelle la variance est égale à la moyenne. La transformation racine carrée permet alors assez bien de normaliser la

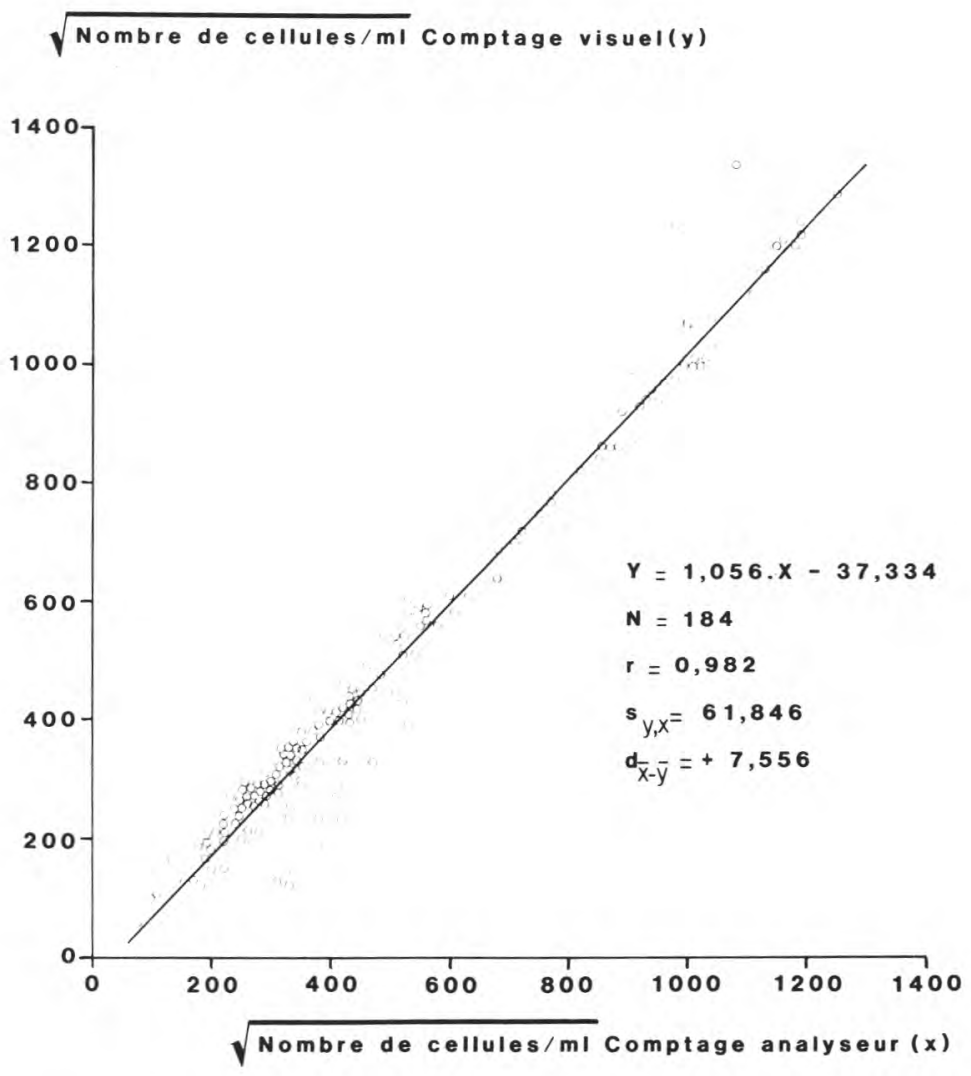

Fig. 5. Relation entre les résultats de numération des cellules somatiques du lait cru par la technique DEFT à l'aide d'un comptage par analyseur d'image et d'un comptage visuel, après transformation racine carrée des résultats. 
Tableau VI. Relation entre les résultats du comptage visuel (y) et du comptage semi-automatique (x) pour différentes concentrations du lait en cellules somatiques (après transformation racine carrée).

\begin{tabular}{llllll}
\hline \multirow{2}{*}{$\begin{array}{l}\text { Nombre de } \\
\text { cellules/ml }\end{array}$} & \multirow{5}{4}{ Paramètres statistiques } \\
\cline { 3 - 6 } & $N$ & $r$ & Equation de régression & $\mathrm{s}_{\mathrm{y}, \mathrm{x}}$ & $\mathrm{d}_{\overline{\mathrm{x}}-\overline{\mathrm{y}}}$ \\
\cline { 3 - 6 } & & & & & \\
Moins de $10^{5}$ & 66 & 0,607 & $y=0,474 x+103,653$ & 50,35 & $+35,09 *$ \\
$10^{5}-5.10^{5}$ & 66 & 0,908 & $y=0,976 x+8,112$ & 43,86 & $+3,02$ \\
Plus de $5.10^{5}$ & 52 & 0,902 & $y=1,069 x-45,393$ & 70,28 & $-21,64^{*}$ \\
& & & & & \\
\hline
\end{tabular}

$\mathrm{N}$ : nombre d'échantillons; ${ }^{*}$ et ${ }^{*}$ : différence entre les moyennes $\bar{x}$ et $\bar{y}$ significativement $>$ ou $<0$, respectivement au seuil de 5 et $1 \%$.

Tableau VII. Répétabilité du comptage visuel et du comptage par analyse d'image en fonction du niveau de concentration cellulaire des échantillons de lait.

\begin{tabular}{|c|c|c|c|c|c|c|c|}
\hline \multirow{2}{*}{$\begin{array}{l}\text { Nombre de } \\
\text { cellules/ml }\end{array}$} & \multirow[b]{2}{*}{$N$} & \multicolumn{3}{|c|}{ Comptage visuel } & \multicolumn{3}{|c|}{ Comptage analyseur } \\
\hline & & $\mathrm{s}_{\mathrm{r}}(\log )$ & $\mathrm{s}_{\mathrm{r}}(\sqrt{)}$ & $R S D^{+}$ & $\mathrm{s}_{\mathrm{r}}(\log )$ & $s_{r}(\sqrt{)}$ & $R S D^{+}$ \\
\hline Moins de $10^{5}$ & 66 & 0,112 & 26,5 & 29,4 & 0,065 & 15,1 & 16,1 \\
\hline $10^{5}-5.10^{5}$ & 66 & 0,055 & 28,1 & 13,5 & 0,038 & 19,1 & 9,1 \\
\hline Plus de $5.10^{5}$ & 52 & 0,024 & 28,4 & 5,7 & 0,022 & 26,3 & 5,2 \\
\hline Total & 184 & 0,076 & 27,6 & 19,1 & 0,046 & 20,2 & 11,2 \\
\hline
\end{tabular}

$N$ : nombre d'échantillons; $s_{r}(\log )$ : écart type de répétabilité $\left(\right.$ en $\left.\log _{10}\right) ; s_{r}(V)$ : écart type de répétabilité (en racine carrée); $\mathrm{RSD}^{+}$: écart type relatif supérieur de répétabilité (en données non transformées) calculé à partir de $\mathrm{s}_{\mathrm{r}}(\mathrm{log})$.

distribution à tous les niveaux de concentration cellulaire du lait (Fig. 5 et Tableau VI).

Le coefficient de régression de l'équation de calibrage (Fig. 5) est significativement $>1$, avec une erreur standard $s_{b}$ de 0,015 ; en revanche, les moyennes $\sqrt{(\text { comptage visuel })}=526,91$ et $\sqrt{(\text { comptage }}$ analyseur $)=534,46$ ne sont pas statistiquement différentes à $P<0,05$. II est donc nécessaire d'appliquer aux résultats obtenus avec l'analyseur d'image l'équation de calibrage de la Figure 5, pour corriger ces résultats du défaut de calibrage. D'autre part sur le Tableau VI, on observe, en moyenne, une surestimation significative des résultats obtenus par ana- 
lyse d'image pour les échantillons contenant moins de $10^{5}$ cellules $/ \mathrm{ml}$; à l'inverse, pour les fortes concentrations cellulaires, le comptage automatique des membranes conduit en moyenne à une sousestimation significative des résultats.

Le Tableau VII présente la répétabilité du comptage visuel et du comptage par analyse d'image des cellules somatiques sur membranes DEFT, calculée après transformation logarithmique ou transformation racine carrée des données. Conformément à ce qui a été observé pour l'étude de la justesse, la transformation racine carrée permet d'obtenir un écart type de répétabilité sensiblement identique à tous les niveaux de concentration cellulaire du lait. D'autre part, on note des écarts types identiques pour les 2 modes de comptage, sauf pour les échantillons contenant moins de $10^{5}$ cellules $/ \mathrm{ml}$; cela entraîne un RSD+ moyen du comptage visuel moins satisfaisant que celui du comptage automatique : $19,1 \%$ contre $11,2 \%$.

Incidence de la conservation des échantillons à $4^{\circ} \mathrm{C}$ sur les résultats de la technique DEFT

On a observé une baisse significative (à $P$ $<0,05$ ) des nombres de cellules obtenus avec la technique DEFT à partir de 4 jours de stockage à $4{ }^{\circ} \mathrm{C}$ (test $t$ par paires) (Dagnelie, 1970); en effet, sans addition de conservateurs, les nombres de cellules sont passés, en moyenne, de 249000 à 218000 après 4 jours de conservation à $4^{\circ} \mathrm{C}$. En revanche, avec addition d'un des 3 conservateurs testés, nous n'avons mis en évidence aucune variation significative des nombres de cellules en 15 jours de stockage.

\section{DISCUSSION ET CONCLUSION}

Notre étude confirme l'intérêt de la technique DEFT pour la numération des cellules somatiques du lait cru (Pettipher \& Rodrigues, 1981), en précisant ses principales caractéristiques de fidélité et de justesse. La justesse obtenue dans notre essai pour la DEFT comme pour l'appareil Fossomatic ne peut être comparée à celle donnée dans les études antérieures, soit parce que les auteurs se sont limités au calcul du coefficient de corrélation (Pettipher \& Rodrigues, 1981), soit parce que les calculs ont été effectués sans transformation ou après transformation racine carrée des données (Grappin \& Jeunet, 1974; Schmidt Madsen, 1975).

Avec un écart type résiduel $s_{y, x}$ d'environ $0,08 \log _{10}$ cellules $/ \mathrm{ml}$, la technique DEFT présente une précision d'estimation satisfaisante et identique à celle de l'appareil automatique Fossomatic. Le défaut de calibrage est lié à une légère sousestimation du nombre de cellules qui peut être attribuée à diverses causes : la lyse de certaines cellules en raison du traitement au triton $X 100$, ou de l'application d'une dépression trop forte lors de la filtration, l'absence de coloration de certaines cellules, la présence d'amas dans les échantillons de fortes concentrations cellulaires ou encore de débris qui rendent le comptage parfois difficile.

La répétabilité de la technique DEFT est moins satisfaisante que celle annoncée par Pettipher \& Rodrigues (1981); cependant, le petit nombre d'échantillons analysés dans leur étude ne permet pas d'estimer avec suffisamment de certitude la fidélité de la technique. En revanche, dans le cas de la technique de référence, la répétabilité obtenue au cours de notre essai est assez proche de celle trouvée par Thompson et al. (1976) ou Read et al. 
(1971). Quant à celle de l'appareil Fossomatic $\left(\mathrm{RSD}^{+}=5,4 \%\right.$ ), elle semble légèrement supérieure à celle obtenue par Grappin \& Jeunet (1974) ou Schmidt Madsen (1975); le mode de calcul différent, des niveaux de concentration différents et surtout une amélioration de l'appareillage depuis 15 ans en sont probablement à l'origine.

En outre, contrairement à la majorité des études précédentes, nous avons tenté d'évaluer la part, dans la variance de reproductibilité, des différentes phases suivies pour les techniques microscopiques. Des auteurs, comme Thompson et al. (1976), ont déjà abordé cette étude pour la technique de référence mais en se limitant à un petit nombre d'échantillons de lait et sans effectuer de transformation des données. En accord avec ce qui avait été observé pour la numération de la flore totale du lait (Dasen et al., 1987), cette étude a mis en évidence la rôle majeur de l'opérateur préparant les membranes et de la répétition du comptage sur les résultats de la technique DEFT. Pour la méthode de référence, le protocole expérimental n'a pas permis de tester l'influence de l'opérateur mais comme dans le cas précédent, la répétition du comptage apparaît comme un facteur déterminant de la fidélité. Le rôle très important du comptage peut probablement être attribué à la variabilité du nombre de cellules par champ, surtout lorsque ce nombre est faible (Kirchman et al., 1982); l'augmentation du nombre de champs examinés pour les faibles niveaux n'est d'ailleurs pas suffisante pour uniformiser la variance de répétabilité des techniques microscopiques selon la concentration cellulaire de l'échantillon. D'autre part, dans le cas de la DEFT, on peut également évoquer la présence d'amas ou une mauvaise répartition des cellules à la surface de la membrane, comme cela a déjà été montré pour les bactéries (Jones \&
Simon, 1975). En revanche, pour la technique de référence, la répartition des cellules sur la lame a rarement été évoquée comme facteur déterminant (Duitschaever et al., 1967; Smith, 1969; Ward \& Postle, 1970).

La technique DEFT apparaît comme une technique juste mais peu fidèle, comme la plupart des techniques microscopiques. L'augmentation du nombre de champs analysés serait probablement un moyen efficace pour améliorer sa fidélité mais la fatigue de l'opérateur au microscope impose alors une automatisation du comptage. Cependant, si la fidélité de l'analyseur d'image testé dans notre étude est très satisfaisante, sa précision d'estimation se révèle en moyenne moins bonne que pour le comptage des bactéries (Dasen et al., 1987). Cette justesse peu satisfaisante doit être attribuée aux échantillons de faibles concentrations cellulaires en raison du faible nombre de cellules par champ et de la prise en compte de débris colorés. A l'inverse, au-dessus de $10^{5} \mathrm{cel}$ lules $/ \mathrm{ml}$, la précision d'estimation du comptage automatique des cellules est sensiblement identique à celle observée pour le comptage des bactéries. L'élimination de l'excès de colorant à l'aide de tampon $\mathrm{pH} 3$ au lieu de triton $X 100$ conduirait à une décoloration de la plupart des débris mais entraîne une coloration verte des cellules (Pettipher \& Rodrigues, 1981). Enfin, pour les échantillons contenant plus de 500000 cellules/ml, la sous-estimation significative du nombre de cellules par analyse d'image peut être attribuée à la présence d'amas.

Conformément à ce qui avait été observé par Grappin \& Jeunet (1975) dans leur étude sur l'appareil Fossomatic, le stockage à $4{ }^{\circ} \mathrm{C}$ des échantillons de lait sans addition de conservateur conduit très vite à une baisse significative des comptages de cellules par la DEFT. A l'inverse, l'addi- 
tion de conservateurs permet de stocker les échantillons pendant au moins 2 semaines sans modifier ces résultats.

Théoriquement, la distribution des cellules à la surface d'une membrane devrait suivre une loi de Poisson pour laquelle la variance est égale à la moyenne; en effet, nous avons observé que contrairement à la transformation logarithmique, la conversion racine carrée des nombres de cellules permet de normaliser assez bien la distribution autour de la moyenne, à tous les niveaux de concentration cellulaire du lait. En revanche, dès que l'on considère la variabilité des résultats obtenus, par exemple entre préparations différentes, la transformation logarithmique peut se justifier; en outre, elle présente l'avantage, par rapport à une transformation racine carrée, de revenir assez facilement aux données d'origine. Par ailleurs, il semble assez justifié, d'après les résultats de notre étude, de donner une estimation de l'écart type résiduel $s_{y, x}$ qui ne tienne pas compte de l'erreur liée à la répétabilité de la méthode de référence. En effet, avec des techniques comme la DEFT ou l'appareil Fossomatic, cela permet une diminution de la variance résiduelle d'environ $30 \%$; cependant, il faut souligner que cette réduction sera beaucoup plus faible avec la plupart des nouvelles techniques d'appréciation de la qualité bactériologique du lait qui présentent des précisions d'estimation nettement moins satisfaisantes.

En conclusion, pour la numération des cellules somatiques du lait cru, la technique DEFT présente des caractéristiques de fidélité et de justesse intéressantes. Cependant, son développement dans les laboratoires d'usine reste limité, compte tenu du coût élevé des membranes en polycarbonate et de la cadence assez faible.

\section{REMERCIEMENTS}

Les auteurs remercient Monsieur Agnet, Laboratoire Départemental d'Analyses Agricoles de Poligny, pour la réalisation des analyses par le Fossomatic. Ils adressent également leurs remerciements à la société Foss Electric France pour le prêt de l'appareil 4010 et pour l'aide technique qu'elle leur a apportée.

\section{RÉFÉRENCES}

Anonyme (1984) Recommended methods for somatic cell counts in milk. Bull. IDF-FIL 128

Anonyme (1985) Lait. Définition et évaluation de la précision globale des méthodes indirectes d'analyse du lait. Application au calibrage et au contrôle de qualité dans les laboratoires laitiers. Norme provisoire FIL 128

Anonyme (1987) Méthode officielle d'analyse relative au dénombrement des cellules somatiques dans le lait cru. J. O. Repub. Fr. 30.6.87

Armstrong J.A. (1956) Histocherrical differenciation of nucleic acids by means of induced fluorescence. Exp. Cell Res. 11, 640-643

Dagnelie P. (1970) Théorie et Méthodes Statistiques. Vol. 2. Les Méthodes de I'Inférence Statistique. Duculot, Gembloux

Dasen A., Piton C., Grappin R. \& Guerry P. (1987) Evaluation de la technique DEFT associée à un comptage visuel ou à un comptage par analyseur d'image pour la numération de la flore totale du lait cru. Lat 67, 77-95

Duitschaever C.L., Ashton G.C. \& Singh J. (1967) Uniformity of solids on smears of milk containing glycine-2-14C and its relation to cell distribution. J. Dairy Sci. 51, 660-664

Grappin R. \& Jeunet R. (1974) Premiers essais de l'appareil Fossomatic pour la détermination automatique du nombre de cellules du lait. Lait $54,627-644$

Grappin R. \& Jeunet R. (1975) Conditions d'utilisation des méthodes automatiques de dé- 
nombrement des cellules du lait : étalonnage et conservation des échantillons de lait. Lait 55, 650-668

Jones C.G. \& Simon B.M. (1975) An investigation of errors in direct counts of aquatic bacteria by epifluorescence microscopy, with reference to a new method of dyeing membrane filters. $J$. Appl. Bacteriol. 39, 317-329

Kirchman D., Sigda J., Kapuscinski R. \& Mitchell R. (1982) Statistical analysis of the direct count method for enumerating bacteria. Appl. Environ. Microbiol. 44, 376-382

Pettipher G.L. \& Rodrigues U.M.(1981) Rapid membrane filtration epifluorescent microscopic technique for the direct enumeration of somatic cells in fresh and formalin-preserved milk. $J$. Dairy Res. 48, 239-246

Pettipher G.L. \& Rodrigues U.M. (1982) Semiautomated counting of bacteria and somatic cells in milk using epifluorescence microscopy and television image analysis. J. Appl. Bacteriol. 53, 323-329

Pettipher G.L., Mansell R., Mac Kinnon C.H. \& Cousins C.M.(1980) Rapid membrane filtrationepifluorescent microscopy technique for direct enumeration of bacteria in raw milk. Appl. Environ. Microbiol. 39, 423-429

Read R.B. Jr, Bradshaw J.G. \& Peeler J.T. (1971) Collaborative study of confirmatory testing procedures for somatic cells in milk. J. Milk Food Technol. 34, 285-288

Schmidt Madsen P. (1975) Fluoro-optoelectronic cell-counting on milk. J. Dairy Res. 42, 227-239

Smith J.W. (1969) Development and evaluation of the direct microscopy somatic cell count (DMSCC) in milk. J. Milk Food Technol. 32, 434-441

Thompson D.R., Packard V.S. \& Ginn R.E. (1976) Electronic somatic cell count. Chemical method, DMSCC and WMT tests for raw milk. J. Milk Food Technol. 39, 854-858

Von Bertalanffy L. \& Bickis I. (1956) Identification of cytoplasmic basophilia (ribonucleic acid) by fluorescence microscopy. J. Histochem. Cytochem. 4, 481-493

Ward G.E. \& Postle D.S. (1970) Studies using the direct microscopic somatic cell count. J. Milk Food Technol. 33, 389-394 